\title{
Optic Disc Drusen in a Child Diagnosed with Alport Syndrome-Case Report
}

\author{
Adamu Sambo', Mona Aslam², Syam Padmanabha² \\ ${ }^{1}$ University Hospital of Leicester, Leicester, UK \\ ${ }^{2}$ Peterborough City Hospital, Peterborough, UK \\ Email: samboadamu@hotmail.com
}

Received 20 October 2014; revised 26 November 2014; accepted 10 December 2014

Copyright (C) 2014 by authors and Scientific Research Publishing Inc.

This work is licensed under the Creative Commons Attribution International License (CC BY). http://creativecommons.org/licenses/by/4.0/

(c) (i) Open Access

\begin{abstract}
Optic disc drusen are eye abnormalities characterised by calcific degeneration affecting some axons of the optic nerve. Alport syndrome is a collagen IV related nephropathy with well-described pathognomonic ocular features. We present the case of a child who following series of investigations was found to have bilateral optic disc drusen, and eventually a further diagnosis of Alport syndrome confirmed. Literature is clear on the underlined aetiology responsible for both renal and extra renal abnormalities of Alport syndrome, which is not related to development of optic disc drusen. The case described makes it pertinent that not only the associated eye signs of Alport syndrome are monitored, but also early detection of other possible co-existing diseases that may influence outcomes.
\end{abstract}

\section{Keywords}

\section{Alport Syndrome, Optic Disc Drusen, Pseudopapilloedema}

\section{Introduction}

Alport syndrome is a nephropathy resulting from abnormalities affecting $\alpha 3,4$ and 5 chains of type IV collagen [1]; and is characterised by renal and eye symptoms, sensorineural deafness and a positive family history of renal impairment. There is an underlined mutation in the collagen biosynthesis genes COL4A3, COL4A4, and COL4A5 [1] [2]. The result of this disruption is a defective type IV collagen network, which forms an important structural component of basement membranes in the kidney, ears, and eyes [2]-[4]. The mode of inheritance makes a positive family history of renal disease an important diagnostic factor, with X-linked inheritance pattern accounting for over $80 \%$ of cases and is due to mutation involving the COL4A5 gene [1] [3]. The clinical features vary in those cases $(10 \%-20 \%)$ resulting from autosomal recessive and the rarer autosomal dominant pat- 
terns, and are as a result of mutations in COL4A3 and COL4A4 respectively [1].

Anterior lenticonus is pathognomonic of Alport Syndrome [4]. It is a rare condition in which the lens has a conical protrusion of its anterior cortex [5] as a result of defective type IV collagen and is seen in $15 \%$ - $20 \%$ of those with X-linked pattern of the condition [4]. The commonest of the eye abnormalities however is the dotand-fleck retinopathy that is seen in $85 \%$ of X-linked males [3]. Optic disc drusen are however acellular calcific deposits occurring in small and crowded optic discs with abnormal vasculature, and evidence suggests axoplasmic transport alteration and axonal degeneration are involved in its formation [6] [7].

This is a case report of Alport syndrome that was confirmed following a protracted period where investigations for papilloedema initially lead to a diagnosis of optic disc drusen in a child. To the best of our knowledge this is not an eye abnormality known to be associated with Alport syndrome. Optic disc drusen is not an uncommon eye problem [8], and though difficult to diagnose in childhood, towards end of adolescence it becomes more easily identifiable with one of the clinical presentations being visual field loss [9].

\section{Case Report}

This 15-year-old boy first presented to the orthoptist at one year of age with concerns of left sided convergent squint that was confirmed on examination. He was referred to the optician at that time for hypermetropic correction, and treatment with eye occlusion and glasses were commenced. At four years of age there were new concerns of poor vision and ophthalmology examination revealed visual acuity of 6/12 and 2/60 on the right and left eyes respectively. An elevated optic disc on the right eye was also noted, while the left disc appeared pale and crowded. In addition to these eye findings, the optician was concerned the child was obese. To rule out optic chiasmal syndrome, an MRI of the orbit and brain was performed which was reported as normal. Ophthalmology management involving treatment for hypermetropia was subsequently continued.

Two years later he had an electroretinogram done which confirmed pseudopapilloedema with decreased patterns on visual evoked potentials. No abnormality was evident on a repeat MRI despite worsening visual symptoms and latest ophthalmologic findings. However, the child's previous history was suggestive of on-going intermittent haematuria, for which he was then referred to a paediatrician. The paediatrician established a positive history of maternal haematuria in teenage years, along with multiple kidney biopsies that were consistently reported normal. Whilst an older sibling of the child had an unidentified visual problem, there were however no other family members with history of kidney disease. Microscopic haematuria and significant proteinuria were also identified on routine urinalysis in the child. Normal C3 and C4 levels were reported, as well as negative screens for antinuclear antibodies (ANA), anti-neutrophil cytoplasmic antibodies (ANCA) and anti-tubular basement membrane antibodies.

Ophthalmology review after another couple of years revealed bilateral disc elevation that was worse on the right eye, and visual acuity of 6/6 and 6/60 on right and left eyes recorded respectively. Ultrasound of eyes revealed bilateral optic disc drusen as the cause for his pseudopapilloedema. He was referred back to the paediatric team, as there were issues around missed appointments. Persistent proteinuria (protein creatinine ratio 155 $\mathrm{g} / \mathrm{mol}$ ) with normal renal function (eGFR $109 \mathrm{ml} / \mathrm{min} / 1.73 \mathrm{~m}^{2}$ ) and blood pressure, and subsequent evaluations by a paediatric nephrologist led to a kidney biopsy being performed at 12 years of age. Light microscopy and electron microscopy were consistent with a progressive hereditary nephritis picture, but no pathognomonic features were identified. Involvement of a geneticist in view of suspicion for Alport syndrome resulted in COL4A mutation analysis, which confirmed hemizygous deletion of exons 3 to 13 on COL4A5.

The child currently is being followed up by a team of multiprofessionals, with management of obesity amongst the key aims. He is currently on enalopril for the proteinuria (latest protein creatinine ratio $243 \mathrm{~g} / \mathrm{mol}$ ) with his most recent renal function reflective of some chronic renal impairment (eGFR $79 \mathrm{ml} / \mathrm{min} / 1.73 \mathrm{~m}^{2}$ ). New concerns of recurrent headaches with vomiting have resulted in a repeat head MRI that is reassuringly normal. Serial assessments have not identified concerns as regards to hearing impairment.

\section{Discussion}

The child described in the report reflects a classical case of Alport syndrome, in terms of presentation and course towards diagnosis. A background of haematuria in the child and maternal history of renal disease, supported by findings on urinalysis was instrumental towards diagnosis. Microscopic haematuria is the commonest of renal symptoms [2] and is seen in $100 \%$ of X-linked cases. Other renal symptoms that develop with advancing age are 
proteinuria, hypertension, and signs of chronic renal failure [1] [4]. Bilateral high frequency sensorineural hearing loss typically occurs in late childhood or early adolescence in males with X-linked mode of the disease, which correlates with this case.

The unfamiliar feature was that of a prolonged history of visual deterioration with late diagnosis of drusen, a condition that may potentially have serious long-term implications on vision. Although the current available evidence shows anterior lenticonus to be pathognomonic of Alport syndrome [4], the presence of two other ocular abnormalities is strongly suggestive of the condition; these are dot and fleck retinopathy, and posterior polymorphous corneal dystrophy [10] [11]. The "lozenge" or "dull macular reflex" appearance suggests severe confluent perimacular dot and fleck retinopathy; a sign associated with early onset renal failure [12]. The defective collagen basement membrane affecting the retina, lens and cornea explains the aforementioned eye abnormalities. However, despite the early onset of visual concerns and serial eye examinations, none of these associated eye abnormalities were identified. Optic disc drusen is thought to be the result of pathology at the level of the optic nerve head itself [8]. It is therefore unlikely that the pathophysiological processes of the two conditions are associated.

Interestingly, optic disc drusen and the well-documented ocular abnormalities of Alport syndrome share the tendency for late diagnosis. Optic disc drusen is not easily identifiable in children as mostly the drusen are usually buried within the disc [6], are asymptomatic, vary in size and are undetectable by fundoscopy. The only sign in some cases is a mild or moderate elevation of the optic disc that may represent true papilloedema or pseudopapilloedema [6] [13]. A case involving elevated optic disc identified at 5 years of age that developed faint signs of drusen at 12 years had been documented [9]; with progression from anomalous optic discs to ophthalmoscopically visible optic nerve drusen occurring over a 16-year period. In the case described here, a unilateral elevated optic disc was noted as early as three years of age, but the diagnosis of bilateral drusen was confirmed at nine years of age, following series of eye examinations and 2 MRI scans. It seems there are no specific early signs for drusen other than unexplainable clinical features like pseudopapilloedema and other developmental optic nerve anomalies that are noticed incidentally on routine ophthalmoscopy examinations [8]. A recent case report has shown that optic disc drusen has a greater tendency to form in eyes with a small scleral canal, and it would therefore seem probable to find a higher rate of the condition in hypermetropic eyes [14] [15]. The child in this case had hypermetropia needing incremental correction throughout his childhood. Further research in this area is needed to demonstrate an association.

An observation has also been described between visible optic disc drusen and decreased visual acuity with loss of peripheral visual field. Though patients with buried optic disc drusen have not been well characterised, a review of patients with the condition suggests that clinical symptoms correlate to visibility of the drusen [16]. Concerns of deteriorating vision in the child prompted serial assessments until the time when drusen was visibly confirmed. This means diagnosis can hugely depend on late identification of those with visual drusen as in this case, despite knowledge that a progressive buried phenomenon of the disease may have been present for some time. It is debatable however if early identification will alter progression of the condition. The visual field loss can occur in patients with longstanding optic disc drusen, with other associated rare problems such as ischaemic complications reported [7] [8]. The prognosis for a patient similar to this case with optic disc drusen and Alport syndrome is not clear, but can be expected to have a huge impact on vision.

This case therefore raises a number of discussions surrounding early detection and management of optic disc drusen and possible association with Alport syndrome. Evidence from research will be required to support early investigation for buried drusen based on suspicion of pseudopapilloedema. Ultrasounds in addition to newer modalities such as optic nerve tomography are proving useful in the diagnosis of drusen [8], as applied in this reported case.

\section{Conclusion}

The outcome for both conditions without doubt necessitates need for early diagnosis and monitoring for complications. Though our current knowledge of optic disc drusen suggests an aetiology that is different to that of ocular abnormalities commonly seen in Alport syndrome, this case highlights the need for consideration of other eye abnormalities in the syndrome. Whether the clinical finding of optic disc drusen should prompt clinical evaluation of inherited renal disease like Alport syndrome, is an issue that needs further research into. Furthermore, the case poses as a reminder to optic disc drusen being a very important differential for papilloedema, and 
its consideration may yet prevent delayed diagnosis and aid appropriate use of investigation resources.

\section{References}

[1] Rees, L., Webb, N.J. and Brogan, P.A. (2007) Paediatric Nephrology. Oxford University Press, New York.

[2] Saxena, R. and Devaragen, P. (2014) Alport Syndrome. http://emedicine.medscape.com/article/981126-overview

[3] Saxena, R. and Devaragen, P. (2014) Alport Syndrome Clinical Presentation. http://emedicine.medscape.com/article/238260-clinical

[4] Kashtan, C.E. (2010) Collagen IV-Related Nephropathies (Alport Syndrome and Thin Basement Membrane Nephropathy). In: Pagon, R.A., Bird, T.D., Dolan, C.R. and Stephens, K., Eds., Gene Reviews [Internet] Seattle (WA), University of Washington, Seattle.

[5] Blaise, P., Delanaye, P., Martalo, O., Pierard, G.E., Rorive, G. and Galand, A. (2003) Anterior Lenticonus: Diagnostic Aid in Alport Syndrome. Journal Francais d Ophtalmologie, 26, 1075-1082.

[6] Lam, B.L., Morais Jr., C.G. and Pasol, J. (2008) Drusen of the Optic Disc. Current Neurology and Neuroscience Reports, 8, 404-408.

[7] Khonsari, R.H., Wegener, M., Leruez, S., Cochereau, I. and Milea, D. (2010) Optic Disc Drusen or True Papilledema? Revue Neurologique (Paris), 166, 32-38. http://dx.doi.org/10.1016/j.neurol.2009.05.003

[8] Davis, P.L. and Jay, W.M. (2003) Optic Nerve Head Drusen. Seminars in Ophthalmology, 18, 222-242. http://dx.doi.org/10.1080/08820530390895244

[9] Spencer, T.S., Katz, B.J., Weber, S.W. and Digre, K.B. (2004) Progression from Anomalous Optic Discs to Visible Optic Disc Drusen. Journal of Neuro-Ophthalmology, 24, 297-298. http://dx.doi.org/10.1097/00041327-200412000-00006

[10] Savige, J. and Colville, D. (2009) Opinion: Ocular Features Aid the Diagnosis of Alport Syndrome. Nature Reviews Nephrology, 5, 356-360. http://dx.doi.org/10.1038/nrneph.2009.65

[11] Colville, D.J. and Savige, J. (1997) Alport Syndrome. A Review of Ocular Manifestations. Ophthalmic Genetics, 18, 161173. http://dx.doi.org/10.3109/13816819709041431

[12] Colville, D., Wang, Y.Y., Tan, R. and Savige, J. (2009) The Retinal “Lozenge” or “Dull Macular Reflex” in Alport Syndrome May Be Associated with a Severe Retinopathy and Early-Onset Renal Failure. British Journal of Ophthalmology, 93, 383-386. http://dx.doi.org/10.1136/bjo.2008.142869

[13] Gossman, M.V. and Giovannini, J. (2014) Pseudopapilledema. http://emedicine.medscape.com/article/1217393-overview\#a0104

[14] Strassman, I., Silverston, B., Seelenfreund, M., Landau, L., Scher, A. and Berson, D. (1991) Optic Disc Drusen and Hypermetropia. Metabolic, Pediatric, and Systemic Ophthalmology, 14, 59-61.

[15] Memon, G.M., Zafar, S., Shakir, M., Kamil, Z. and Bokhari, S.A. (2012) Bilateral Optic Disc Drusen in Hypermetropic Children of a Family. Pakistan Journal of Ophthalmology, 28, 163-165.

[16] Wilkins, J.M. and Pomeranz, H.D. (2004) Visual Manifestations of Visible and Buried Optic Disc Drusen. Journal of Neuro-Ophthalmology, 24, 125-129. http://dx.doi.org/10.1097/00041327-200406000-00006 
Scientific Research Publishing (SCIRP) is one of the largest Open Access journal publishers. It is currently publishing more than 200 open access, online, peer-reviewed journals covering a wide range of academic disciplines. SCIRP serves the worldwide academic communities and contributes to the progress and application of science with its publication.

Other selected journals from SCIRP are listed as below. Submit your manuscript to us via either submit@scirp.org or Online Submission Portal.
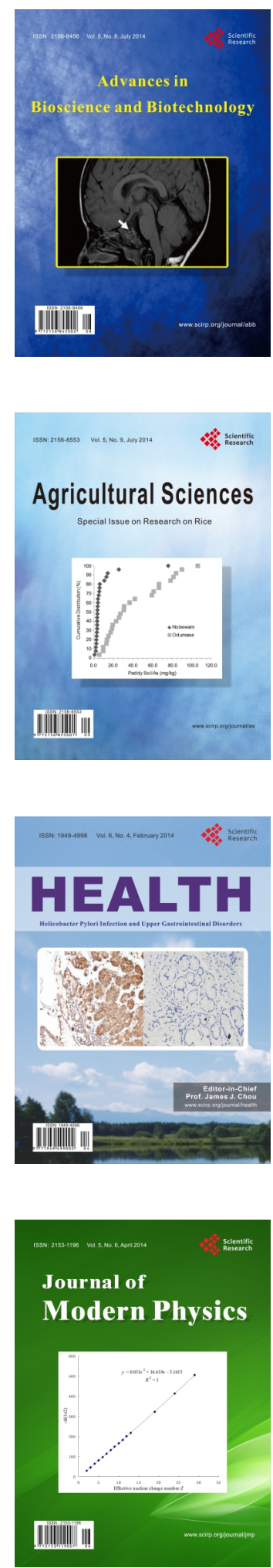
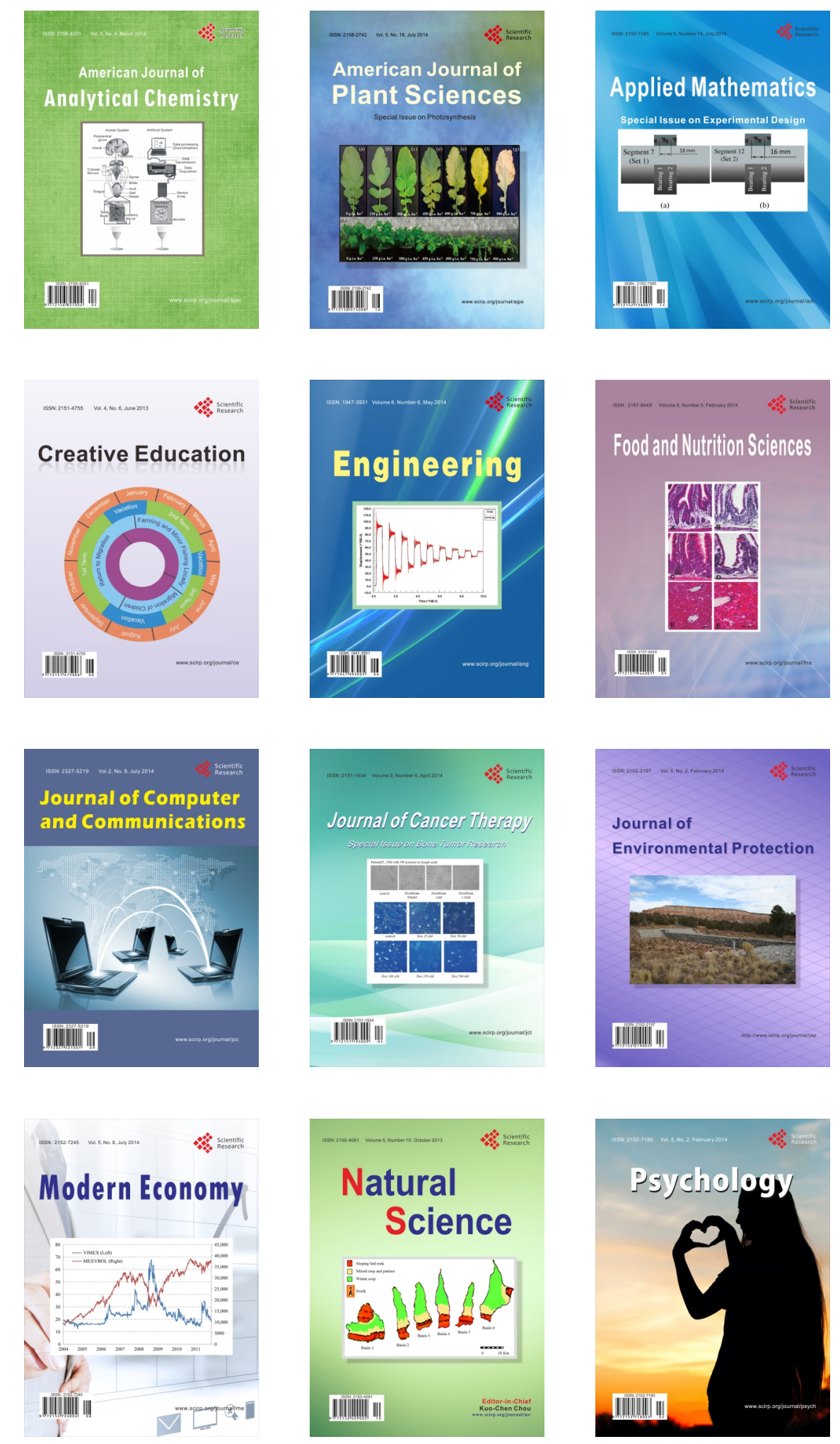